\title{
Quantitation of Motion Perception in the Digits: A Psychophysical Study in Normal Human Subjects
}

\author{
Emre Kokmen, MD, Robert W. Bossemeyer, Jr, MScEE, and William J. Williams, PhD
}

\begin{abstract}
Threshold perception of motion of the digits was obtained in 14 normal subjects. The metacarpophalangeal joint of the index and the fifth finger of each hand and the metatarsophalangeal joint of the hallux of each foot were passively moved up and down with respect to a horizontal plane defined by the palmar or plantar surface. The motion was sinusoidal at frequencies of 0.5 and $5.0 \mathrm{~Hz}$. A modified von Békésy paradigm similar to that used in audiometry was utilized to yield threshold levels of motion sensation. There was little difference in the thresholds obtained for the different joints. The difference between high- and low-frequency stimulation, however, was significant $(p<0.001)$ : the $0.5 \mathrm{~Hz}$ threshold was found to range from 0.8 to 1.0 degree, whereas the $5.0 \mathrm{~Hz}$ threshold varied from 0.4 to 0.6 degree. It is thought that motion sense is largely dependent on joint receptor contributions, but muscle and cutaneous receptors may also contribute to this proprioceptive sensation.
\end{abstract}

Kokmen E, Bossemeyer RW Jr, Williams WJ: Quantitation of motion perception in the digits: a psychophysical study in normal human subjects. Ann Neurol 2:279-284, 1977

A number of efforts have been made in the past to quantitate somatic senses [5], including a few studies which have attempted to measure perception of motion of limbs or digits. In 1889 Goldscheider [8] recorded perception of passive motion in several joints moved with an elaborate hydraulic system. Laidlaw and Hamilton [14] moved joints passively with a hand-cranked mechanical device. Browne, Lee, and Ring [2] moved the metatarsophalangeal joint (MTP) passively with a motor-driven assembly. The subjects indicated perception by depressing a switch, causing the angle through which the digit had been moved to be recorded. Provins [20] used a similar method to study the appreciation of index finger movements at the metacarpophalangeal (MCP) joint.

A quantitative psychophysical evaluation is needed to study the characteristics of motion perception in humans. Bedside clinical sensory testing methods lack precise control of stimulation delivery and response recording and evaluation. The intensity, frequency, direction, and sites of application of the stimuli must be controlled, and responses of the subjects must be recorded consistently.

We have devised a minicomputer-assisted method of assessing perception of passive sinusoidal motion of the MCP and MTP joints in man, utilizing a modified von Békésy procedure similar to that used in audiometry [22]. We believe that a reliable and precise quantitation technique is necessary as a first step in studying the nature of proprioceptive sensations in humans. Such a merhod can also be a useful tool in the longitudinal and comparative evaluation of patients with selected neurological conditions as well as in assessing sensory performance of selected populations, such as the aged versus the young. However, before the method can be used in clinical studies, some normative values must be established. We report the motion perception thresholds obtained in a group of young, healthy naive subjects.

\section{Methods and Materials}

An electrically controlled mechanical system was developed to move the fingers and toes up and down and to articulate the MCP joint of the index and fifth finger of each hand and the MTP joint of the hallux of each foot. In all cases, the subject was completely relaxed and the movement of the digit was passive. The finger or toe was placed in a padded plastic clamp that held the digit firmly and prevented motion of the distal interphalangeal joint. Since we were concerned at the outset that differential pressures on the dorsal or ventral surface of the digit might give motion clues, several types of finger and toe holders, including one with an inflatable inner liner, were tried in preliminary experiments. The final model used in this study applied uniform pressure to the digit on all sides via an intermediate liner fabricated from a silicon gel material.

A minicomputer was programmed to run the experiment as well as to record and sort data. An identifying number; the subject's age, sex, and handedness; the joint to be tested; the date; and the number of trials used were entered by tele-
From the Departments of Neurology and of Electrical and Computer Engineering, University of Michigan Medical Center, Ann Arbor, MI.
Accepted for publication May 20, 1977.

Address reprint requests to Dr Kokmen, University of Michigan Medical Center, Ann Arbor, MI 48109. 
typewriter and stored in computer disc files. The threshold stimulus intensity in degrees, the frequency of stimulation, and summary statistics concerning the response of the subjects were also stored in the disc file. A block diagram of the system is shown in Figure 1.

Fourteen normal test subjects, 6 women and 8 men, were recruited from co-workers, acquaintances, and students. The age range was from 18 to 29 years with a median of 23 and a mean of 22.2 years. Thirteen subjects were righthanded and 1 was left-handed. This was the first testing of this type for each of the subjects; personnel who had been involved in the development of the apparatus and had tested and retested themselves were excluded from the study group.

The subjects were seated comfortably in a dimly lit room. They extended their extremity to be tested into the apparatus (Fig 2). A cloth drape was positioned over the hand or foot to exclude vision. The digit to be tested was isolated from the others by gently applied cloth straps, and the extremity was supported (Fig 2, inset). A standardized paragraph stating the goals and procedures of the experiment was read aloud, and the subject was given the opportunity to ask questions before testing was begun.

The joint to be tested was passively flexed and extended in a sinusoidal fashion; these sinusoidal stimuli were varied in intensity and frequency. The digit to be tested was on the same plane as the palmar surface of the hand or the plantar surface of the foot at the beginning of the experiment. For a given frequency, the stimulus intensity gradually increased from zero to a maximum of 10 degrees of total motion of the joint. The sinusoid stimulus was applied continuously and was slowly increased in intensity until perceived by the subject. The subjects were instructed to concentrate on a sense of motion of the digit and were asked to indicate the earliest perception of movement by placing a finger of the other hand on a specially designed response switch. When the subject indicated perception of movement by pressing the response switch, the stimulus amplitude was gradually decreased until it was below the threshold for sensation. The subject was instructed to release the finger from the switch when the sensation of movement was lost, and the amplitude of the stimulus was then increased once more. In this way, it was possible to obtain a series of upper and lower thresholds of sensation of movement for each digit. This is similar to von Békésy's audiometric method [22]. The actual threshold was assumed to be the level of stimulus intensity above which the subject detected movement more than $50 \%$ of the time. Precise measurement of the stimulus intensity was accomplished electronically and recorded by the computer whenever the subject responded by moving his finger to or from the switch.

To acquaint the subject with the testing procedure and the sensations he might be experiencing, a trial test on the right index finger was carried out before data gathering was begun. At the end of the trial test, another conversation session was held with the subject to answer questions or clarify points. The results of this trial test were not included in the final data reported.

For each digit tested, seven pairs of threshold estimates were obtained at test frequencies of 0.5 and $5.0 \mathrm{~Hz}$. The first two pairs were discarded to reduce or eliminate carryover from a previous rest frequency, and the remaining five measurements were used for all calculations. The testing time was approximately 15 minutes for each digit tested, thus requiring approximately 90 minutes for the completion of an experiment for a given subject. This included changeover time between digits.

\section{Results}

The Table gives a sample listing of the data from one digit, including the mean value of upper, lower, and actual thresholds as well as the standard deviations for each frequency tested. A coefficient of variation obtained by multiplying the standard deviation by 100 and dividing the result by the mean is also shown. A listing of each data point is also included.

Fig 1. Diagram of the testing and recording apparatus.

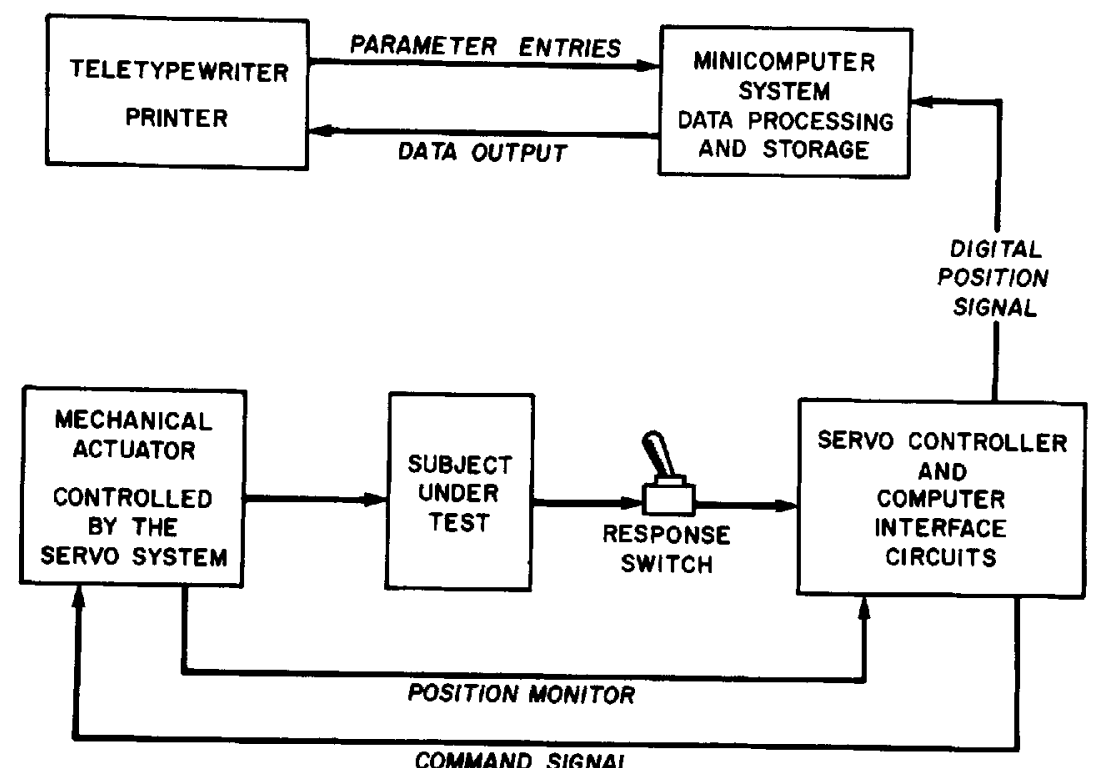

COMMAND SIGNAL 


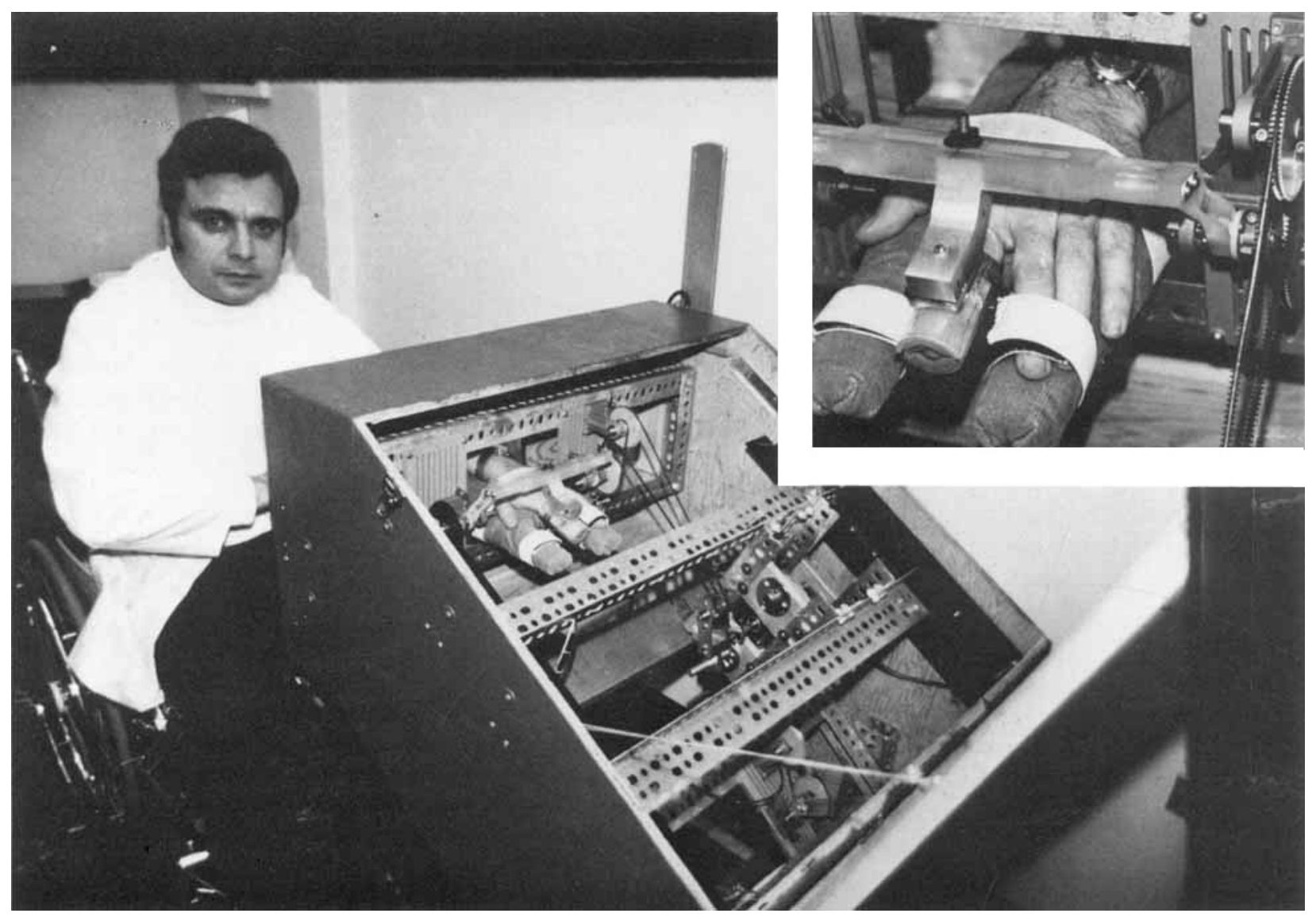

Fig 2. The testing apparatus, showing mechanical assembly. Inset sbows subject's index finger in place.

Sample of Atailable Computer Data for Each Digit Tested

Female

22 years

Right-handed

Right index finger

\begin{tabular}{|c|c|c|c|c|c|c|c|c|c|}
\hline \multirow[b]{2}{*}{ Frequency } & \multicolumn{3}{|c|}{ Upper Threshold } & \multicolumn{3}{|c|}{ Lower Threshold } & \multicolumn{3}{|c|}{ Threshold } \\
\hline & $\mathrm{M}$ & $\mathrm{SD}$ & $\mathrm{CV}$ & M & $\mathrm{SD}$ & $\mathrm{CV}$ & $\mathrm{M}$ & $\mathrm{SD}$ & $\mathrm{CV}$ \\
\hline 0.5 & 1.05 & 0.07 & 6.67 & 0.52 & 0.06 & 11.54 & 0.79 & 0.05 & 6.33 \\
\hline 5.0 & 0.54 & 0.04 & 7.40 & 0.35 & 0.03 & 8.57 & 0.45 & 0.02 & 4.44 \\
\hline
\end{tabular}

Individual data points for the above means for upper and lower thresholds:

Frequency $=0.5 \mathrm{~Hz}$

Response No.

1

2

3

4

5

Frequency $=5.0 \mathrm{~Hz}$

Response No.

\section{1}

2

3

4

5

Upper Thresbold
1.17
1.02
1.00
1.02
1.05

Upper Tbresbold

0.57

0.48

0.52

0.57

0.55
Lower Thresbold

0.57

0.48

0.60

0.52

0.45

Lower Threshold

0.31

0.36

0.36

0.38

0.36

$\mathrm{M}=$ mean, expressed in degrees of peak sinusoidal displaccment of finger; $\mathrm{SD}=$ standard deviation; $\mathrm{CV}=$ coefficient of variation. 


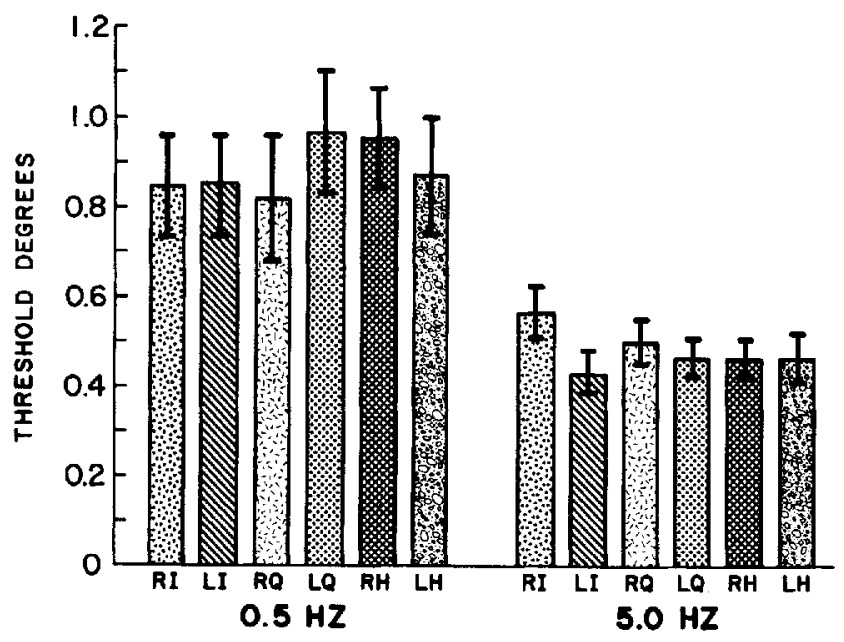

Fig 3. Mean values and standard deviation of average thresbolds in the 14 subjects for each of the six digits tested at 0.5 and $5.0 \mathrm{~Hz}$. ( $\mathrm{RI}=$ right index metacarpopbalangeal joint; $\mathrm{LI}=$ left index metacarpophalangeal joint; $\mathrm{RQ}=$ right quinti metacarpopbalangeal joint; $\mathrm{LQ}=$ left quinti metacarpophalangeal joint; $\mathrm{RH}=$ right hallux metatarsophalangeal joint; $\mathrm{LH}=$ left ballux metatarsophalangeal joint.)

Mean threshold values and standard deviation for six digits of normal subjects at the two stimulation frequencies are shown in Figure 3. The threshold value lies between 0.8 and 1 degree stimulus intensity for each of the six joints tested at $0.5 \mathrm{~Hz}$. At $5.0 \mathrm{~Hz}$ the value is between 0.4 and 0.6 degree of stimulus intensity.

A two-factor analysis of variance was performed on the data to determine if differences between means could be attributed to naturally occurring experimental variation. The difference between high- and lowfrequency thresholds was significant at the $p<0.001$ level. When the thresholds obtained from the six digits at the same frequency were compared, there were no significant differences.

\section{Discussion}

In 1826 Bell [1] proposed that proprioception, the sense of position of limbs in space, is a separate system, and the exact neurophysiological nature of proprioception has been a matter of discussion since then. One of the problems in this area has been the rather vague definition of the terms position sense. motion sense, and kinesthesia. For our purposes we will define position sense as the subjective appreciation of a static or fixed joint sensation, and motion sense or kinesthesia as the subjective appreciation of motion around a joint.
We have elected to use sinusoidal movements of digits as the set of defining stimuli. This, of course, is not the only possible set, but the use of sinusoidal stimuli is attractive in several respects. Such stimuli have been profitably used in other sensory tests, notably in audition and cutaneous sensation [22]. In addition, a large quantity of data are already available on the response of joint receptors to sinusoidal stimuli in animals $[15,24,26]$.

At the most peripheral level, receptors located in joint capsule, muscle, tendon, or skin and subcutaneous tissue may be responsible for initiating the neurophysiological events that will terminate in perception of motion. For many years the view has prevailed that joint receptors more or less exclusively convey proprioception sensation $[16,18]$. Goldscheider [8] observed a blunting of motion sense when electric current was applied to the region of the elbow joint. This led him to postulate that the receptors mediating this sensation must be located in the joint capsule. When Browne, Lee, and Ring [2] injected a local anesthetic into the joint capsule, 8 of their 9 subjects were unable to determine the position of their great toe until extremes of range of movement were reached. Provins [20] anesthetized the proximal finger joint of his subjects and found that the angle of movement required to provoke sensation of motion rose from approximately 4 degrees (control group) to around 15 degrees (anesthetic group). Wyke [25] mentioned patients with difficulties of gait following damage to articular nerves.

The role of receptors located in muscles has been investigated as well. Giaquinto et al [7] found no behavioral or encephalographic changes in sleeping cats when group I muscle afferents were stimulated. Swett and Bourassa [21] were unable to condition cats to respond to stimulation of group I muscle afferents until they stimulated cutaneous afferents. Gelfan and Carter [6] found that pulling on exposed muscle tendons in man failed to evoke sensations of change in muscle tension and joint position. While these findings suggest that muscle receptors do not contribute to motion perception, another set of observations indicates a definite role for muscle afferents in position sense and kinesthesia. In 1901 Pillsbury [19] studied the knee joints with Goldscheider's methods. He noted, however, that sensation was blunted when current was applied to the region of the tendons of the muscles subserving the joints. When muscle associated with a particular joint is vibrated, systematic errors in position and judgment occur, and there is even perception of impossible limb positions [4, 9]. When muscle afferents are preserved but joint afferents are paralyzed by either digital nerve block or joint capsule anesthesia, appreciable kinesthesia and 
position sense remain $[10,12]$. Surgical removal of a joint does not abolish position sense [11].

The role of skin receptors is more difficult to evaluate. Digital nerves supply both skin and joints. We have been unsuccessful in our attempts to abolish cutaneous sensation with topical anesthesia. Moberg [17] has addressed this problem. He states that: "blocking the joint receptors interferes with skin sensation, and blocking cutaneous nerves will include the joint branches. The two factors cannot be separated in this way" [17].

It has been suggested that type II mechanoreceptors in the skin may be able to function as proprioceptors [13]. This type of cutaneous receptor responds readily to skin stretching as well as to pressure on the skin. If cutaneous receptors do contribute to proprioception, type II mechanoreceptors are the most likely candidates for the role. Chambers et al [3] discount the possibility of a proprioceptive role for type II mechanoreceptors on the basis that adjacent units do not always respond in the same way to a particular movement of the limb in cats. When Browne, Lee, and Ring [2] anesthetized the joint capsule of the great toe, the toe could be passed through its full range of movement either upward or downward. The subjects failed to detect movement even when the joint had been moved through its full range. Ultimately, when the extreme of movement was reached, the subjects were able to detect the position of the toe, presumably by means of the sensations generated by skin stretching on the dorsal or volar surface of the joint. If these observations are correct, it would seem that cutaneous receptors could not play a major role in provoking the threshold sensations obtained in our experiments since only small, midrange movements took place. It is not entirely certain that anesthetic action remained exclusively in the joint capsule in the experiments of Browne et al, however.

Our results appear to be quite uniform and reproducible from subject to subject and can be used as a reliable means of measuring motion sense, at least in the joints we tested. The thresholds of motion we obtained are smaller than those reported by Goldscheider, Browne et al, and Provins, who all used constant-velocity measurements. Since we used sinusoidal stimuli, the results should not be comparable in the strictest sense. We found much less variation from subject to subject than did Browne et al.

In studies of various receptor types responding to motion of the hind limb joints of cats, McCall et al [15] found that slowly adapting receptors recorded from the medial articular nerve readily follow sinusoidal stimuli at frequencies from below $0.001 \mathrm{~Hz}$ to at least $7 \mathrm{~Hz}$. The ability of these slowly adapting receptors to follow sinusoidal stimuli increases slowly with fre- quency. Rapidly adapting joint teceptors do not respond to low-frequency sinusoidal stimuli but begin to respond feebly and occasionally at 0.1 to $0.2 \mathrm{~Hz}$ and are quickly provoked into increasingly powerful activity above $1 \mathrm{~Hz}[24,26]$. The rapidly adapting afferents project via the dorsal columns to the nucleus gracilis, whereas present evidence indicates that slowly adapting joint afferents from hind limbs of cats and monkeys, at least, leave the dorsal columns at higher cervical levels $[23,24]$. This may have implications in human perception of joint motion at different frequencies.

Our study does not furnish conclusive data that would clarify the issue of which elements of the peripheral nervous system contribute to perception of motion. However, availability of this reliable, easily tolerated, precise method of quantitation is a necessary first step in psychophysical or psychophysiological studies of kinesthesia.

Supported in part by US Public Health Service Grant NSO 8470.

\section{References}

1. Bell C: On the nervous circle that connects voluntary muscles with the brain. Phil Trans R Soc Lond Part III:163-173, 1826

2. Browne K, Lee J, Ring PA: The sensation of the passive movement at the metatarsophalangeal joint of the great toe in man. J Physiol (Lond) 126:448-458, 1954

3. Chambers MR, Andres KH, Duering MV, et al: The structure and function of the slowly adapting type II mechanoreceptors in hairy skin. J Exp Psychol 57:417-415, 1972

4. Craske B: Perception of impossible limb positions induced by tendon vibration. Science 196:71-73, 1977

5. Dyck PJ: Quantitation of cutaneous sensation in man, in Dyck PJ, Thomas PK, Lambert EF (eds): Peripheral Neuroparhy. Philadelphia, WB Saunders Company, 1975

6. Gelfan S, Carter S: Muscle sense in man. Exp Neurol 18:469473,1967

7. Giaquinto S, Pompeiano O, Swett JE: EEG and behavioral effects of fore- and hindlimb muscular afferent volleys in unrestrained cats. Arch Ital Biol 101:133-148, 1963

8. Goldscheider A: Untersuchungen über den Muskelsinn. Arch Anat Physiol Leipzig Physiol Abteil 1889, pp 369-503

9. Goodwin GM, McCloskey DI, Matthews PBC: Proprioceptive illusions induced by muscle vibration: contribution by muscle spindles to perception. Science 175:1382-1384, 1972

10. Goodwin GM, McCloskey DI, Matthews PBC: The persistence of appreciable kinesthesia after paralyzing joint afferents but preserving muscle afferents. Brain Res 37:326-329, 1972

11. Grigg P, Finerman GA, Riley LH: Joint position sense after total hip replacement. J Bone Joint Surg [Am] 55:1016-1025, 1973

12. Horch KW, Clark FJ, Burgess PR: Awareness of knee joint angle under static conditions. J Neurophysiol 38:1436-1447, 1975

13. Knibestöl M, Vallbö AB: Single unit analysis of mechanoreceptor activity from human glabrous skin. Acta Physiol Scand 80:178-195, 1970

14. Laidlaw RW, Hamilton MA: The quantitative measurement of apperception of passive movement. NY Neurol Inst Bull $6: 145-173,1937$ 
15. McCall WD Jr, Farias MAC, Williams WJ, et al: Static and dynamic responses of slowly adapting joint receptors. Brain Res $70: 221-243,1974$

16. Merton PA: Human position sense and sense of effort. Symp Sor Exp Biol 18:387-.400, 1964

17. Moberg E: Fingers were made before forks. Hand 4:201-206, 1972

18. Mountcastle VB: Medical Physiology, vol 2. St Louis, CV Mosby Company, 1968

19. Pillsbury WB: Does the sensation of movement originate in the joints? Am J Physiol 12:346-353, 1901

20. Provins KA: The effect of peripheral nerve block on the appreciation and execution of finger movements. J Physiol (Lond) 143:55-67, 1958

21. Swetr JE, Bourassa CM: Comparison of sensory thresholds with muscle and cutaneous nerve volleys in the cat. I Neurophysiol 30:530-545, 1967

22. von Békésy G: Experiments in Hearing. New York, McGraw-Hill Book Company, 1960

23. Whitsel BL, Petrucelli LM, Shapiro G: Modality representation in the lumbar and cervical fasciculus gracilis of squirrel monkeys. Brain Res 15:67-78, 1969

24. Williams WJ, BeMent SL, Yin TCT, et al: Nucleus gracilis responses to knee joint motion: a frequency response study. Brain Res 64:123-140, 1974

25. Wyke B: The neurology of joints. Ann R Coll Surg Engl $41: 25-50,1967$

26. Yin TCT, Williams WJ: Dynamic response and transfer characteristics of joint neurons in somatosensory thalamus of the cat. J Neurophysiol 39:582-600, 1976 